\title{
Jost Heintzenberg and Robert J. Charlson (eds): clouds in the perturbed climate system; their relationship to energy balance, atmospheric dynamics, and precipitation.
}

\author{
The MIT Press, Cambridge, Massachusetts, 2009, \\ ISBN 978-0-262-01287-4, 597 pages, USD 40.00 (hardcover).
}

\section{Peter Warneck}

Received: 29 August 2009 / Accepted: 1 September 2009 /

Published online: 23 September 2009

(C) The Author(s) 2009. This article is published with open access at Springerlink.com

Keywords Aerosols $\cdot$ Clouds $\cdot$ Earth's radiation budget $\cdot$ Precipitation $\cdot$ Climate models

This book is the outcome of a discussion meeting held in Frankfurt (Main), Germany, under the sponsorship of the Ernst Strüngmann Forum, which brought together many of the world's leading experts on aerosols, clouds and climate. The result is a thorough review of the current status of the science of clouds. Twenty contributions are loosely divided into four groups of subjects: observations of clouds, related aerosols and their variability; factors that control various types of clouds; the extent and nature of anthropogenic perturbation of clouds; and the understanding and quantification of clouds through modelling. Each group of articles is supplemented by a summary of the discussion sessions related to these topics, and followed by recommendations for future work.

Liquid water and ice contain only a small fraction of total atmospheric water (present in the vapour phase), yet clouds play a significant role in determining the radiation balance of earth and climate in general. While satellite observations now provide a better view on cloud coverage and the associated backscatter of radiation than previous ground-based observations, it remains difficult to understand the factors that control cloud formation and climate-related feedback processes. Two key questions are: How do clouds respond to global warming? And how do clouds respond to anthropogenic aerosols? Currently it is not known how much global warming induces changes in cloudiness and whether the radiative response of clouds will exacerbate, mitigate, or have little effect on the increase in surface temperature. Likewise, it is difficult to quantify changes in the micro-physical and radiative properties of clouds associated with aerosol sulphate resulting from the oxidation of sulphur dioxide produced by combustion of fossil fuels. The book under review deals with these problems as well as with others, but, to repeat the introductory statement, its real value lies

P. Warneck $(\bowtie)$

Max-Planck-Institut für Chemie, 55020 Mainz, Germany

e-mail: biogeo@mpch-mainz.mpg.de 
in the very detailed picture it presents of our current knowledge in this field of the atmospheric sciences. The book also reflects the essence of the Ernst Strüngmann Forum: the identification of important areas that need further study. This aspect was decisive for the success of individual discussion sessions and the meeting as a whole.

Only a fraction of all aerosol particles serve as cloud condensation nuclei $(\mathrm{CCN})$ or ice forming nuclei under conditions of a slight super-saturation of water vapour. The condition for particles to act as $\mathrm{CCN}$ is theoretically well defined for ideal substances, but atmospheric particles consist of a complex mixture of materials, forcing cloud scientists to an empirical treatment. This part of the problem is now better constrained. On the other hand, to achieve a global representation of aerosols and $\mathrm{CCN}$ for inclusion in models will require much more effort. The nature of ice forming nuclei also remains uncertain.

While CCN largely control cloud micro-structure, cloud macro-structure is governed by meteorology, that is, by large-scale atmospheric motions. Boundary layer clouds, which are most ubiquitous, contribute greatly to radiative forcing. Their occurrence shows seasonal variations depending on lower tropospheric stability, but theory is currently insufficiently developed to attribute changes in cloudiness to perturbations in the aerosol burden. Deep convective clouds, in turn, play a key role in atmospheric general circulation. In these systems long- and shortwave cloud forcing nearly cancel each other out. Deep convective clouds cover a wide range of spatial scales that must be bridged computationally but are not, at least not in traditional general circulation models. Factors controlling cirrus clouds comprise both small- and large-scale atmospheric dynamics, ice nucleation behaviour of particles and interactions with terrestrial and solar radiation.

The hypothesis that small particles from air pollution should lead to less efficient formation of precipitation exists for several decades but as the discussion in this book shows, there is no convincing proof that microphysical control of precipitation efficiency has led to reduction in rainfall anywhere on the globe. Extreme rainfall as well as rain from shallow clouds is discussed with a focus on statistical data rather than individual case studies. An additional contribution describes concepts of seeding clouds to increase rainfall and other weather and climate engineering suggestions.

Over the oceans, against a fairly uniform background, satellite observations of aerosol column optical densities clearly show the effect of anthropogenic perturbations. These localized effects, downstream from pollution centres, can be adequately modelled, although the direct radiative effects are underestimated. Global-scale features of aerosol-cloud interactions are also estimated from observations and can be simulated by models over the oceans. On the ground, a network of sun photometers has been used to determine aerosol column optical densities, and these data compare well with remote sensing from space where spatial overlap exists.

Throughout the book it appears indispensible to use abbreviations. Their meaning is not always obvious, but a list of abbreviations is provided for the benefit of the reader. A good subject index and a separate name index complete the volume. Each article is supplemented by copious, up-to-date citations that provide the reader with a broad overview on the current literature. In summary, this unique book can be strongly recommended to all scientists interested in the intricate relations between aerosols, clouds, climate and climate modelling.

Open Access This article is distributed under the terms of the Creative Commons Attribution Noncommercial License which permits any noncommercial use, distribution, and reproduction in any medium, provided the original author(s) and source are credited. 